\title{
Echium montenielluense (Boraginaceae), une nouvelle espèce endémique de Corse
}

\author{
Alain Delage
}

\begin{abstract}
DELAGE, A. (2018). Echium montenielluense (Boraginaceae), a new species endemic to Corsica. Candollea 73: 193-199. In French, English and French abstracts. DOI: http://dx.doi.org/10.15553/c2018v732a3

A plant belonging to the genus Echium L. (Boraginaceae), discovered on the serpentine ridges of Cap Corse is described, characterized morphologically and named Echium montenielluense Delage. Elements relating to its ecology and its demography are presented. Probably related to the Sardinian endemic Echium anchusoides Bachetta et al., the differences between the two taxa are highlighted.
\end{abstract}

\section{Résumé}

DELAGE, A. (2018). Echium montenielluense (Boraginaceae), une nouvelle espèce endémique de Corse. Candollea 73: 193-199. En français, résumés anglais et français. DOI: http://dx.doi.org/10.15553/c2018v732a3

Une plante appartenant au genre Echium L. (Boraginaceae), découverte sur les crêtes serpentiniques du Cap Corse, est décrite, caractérisée morphologiquement et nommée Echium montenielluense Delage. Des éléments relatifs à son écologie et à sa démographie sont présentés. Probablement apparenté à l'endémique sarde Echium anchusoides Bachetta et al., les différences entre les deux taxons sont mises en évidence.

\section{Keywords}

BORAGINACEAE - Echium - Corsica - New species - Taxonomy 


\section{Introduction}

Le genre Echium L. (Boraginaceae) compte six espèces en Corse (JEAnMonod \& Gamisans, 2013). Echium plantagineum L. et E. italicum L. sont communes et largement répandues, tandis que $E$. calycinum Viv. (= E. parviflorum Moench) et $E$. sabulicola Pomel sont rares et localisées. Echium vulgare L. et E. creticum L. ont été observées très rarement, mais nont pas été revues récemment et n'étaient peut-être qu'adventices. Enfin, une septième espèce, $E$. arenarium Guss., indiquée par erreur par Jeanmonod \& Gamisans (2013), n’a jamais été confirmée en Corse, bien qu'elle existe sur la côte nord de la Sardaigne.

Tous ces taxons sont des plantes de basse et moyenne altitude, principalement présentes en dessous de $450 \mathrm{~m}$ et n'excédant pas $700 \mathrm{~m}$ à notre connaissance, poussant en Corse essentiellement dans des pelouses et des formations plus ou moins rudérales. A l'exception d'E. calycinum et E. sabulicola, en conditions normales de végétation, ce sont des végétaux plutôt robustes et à port dressé.

Dans le cadre des activités d'inventaire du Conservatoire Botanique National de Corse (CBNC), pendant nos prospections floristiques sur les crêtes serpentiniques du Capicorsu (Cap Corse), nous avons pu observer en janvier 2016 une petite population d'une Boraginaceae qui nous était inconnue, appartenant vraisemblablement au genre Echium, dont seules les rosettes étaient visibles. Une nouvelle prospection en mai 2017 a permis de confirmer qu'il s'agissait bien d'un Echium qu'il nous a été impossible d'identifier avec certitude en utilisant les flores de référence usuelles pour la Corse (Giв Bs, 1972; Fürnkranz, 1982; VAldés, 2012; JeAnmonod \& Gamisans, 2013; Tison \& De Foucault, 2014; Tison et al., 2014) mais également en confrontant nos échantillons avec un certain nombre de monographies du genre (Giввs, 1971; VALSECCHI, 1977; VALDÉs, 2007).

Cet Echium présente des fleurs à 2 étamines saillantes, à grandes corolles (>10 mm) poilues, ce qui le rapproche du groupe d'E. creticum et $E$. sabulicola mais aussi de l'endémique sarde $E$. anchusoides Bachetta et al. Il pousse entre 900 et 1030 m d'altitude, en milieu naturel non rudéralisé, très peu anthropisé (pâturage bovin très extensif sur les crêtes au-dessus de la localité) et quasiment pas fréquenté à l'heure actuelle. C'est un végétal de taille réduite développant de petites rosettes et des tiges prostrées qui lui confèrent un aspect très inhabituel. Sa présence en altitude, son isolement dans un habitat très particulier, son port et sa floraison rose nous permettent de penser qu'il s'agit d'un taxon nouveau que nous décrivons ci-dessous comme E. montenielluense Delage.

\section{Matériel et méthodes}

La seule population connue, avec env. 250 individus, a été étudiée principalement sur le terrain et sur un très petit nombre d'individus récoltés, compte-tenu de la grande rareté du taxon.
L'analyse morphologique et les caractères donnés dans la description sont basés sur Delage s.n. comprenant 8 tiges fleuries récoltés et conservés au $\mathrm{CBNC}$. Toutefois, cette analyse a été complétée par les caractéristiques des inflorescences, le nombre de fleurs et les longueurs de 20 individus supplémentaires sur le terrain alors que le nombre de ramifications a été mesuré sur 65 tiges. Enfin, les caractéristiques de l'appareil végétatif ont été mesurées sur 36 individus reproducteurs ou présentant une rosette ayant un développement comparable. Nous avons relevé: (a) la longueur des feuilles de la rosette, de l'apex à l'insertion; n'ont pas été mesurées les feuilles du centre de la rosette, dont le développement nétait manifestement pas terminé; (b) la largeur maximale des feuilles; (c) le nombre de tiges fleuries et (d) le nombre et la longueur des ramifications. Nous avons aussi observé l'espèce proche $E$. anchusoides près de Villacidro dans le massif de l'Iglesiente en Sardaigne.

Ces études de terrains ont été complétées par des études bibliographiques, notamment BACHETTA et al. (2000) concernant E. anchusoides et VALDÉs (2012) pour E. creticum et $E$. sabulicola, mais également dans toutes les flores à notre disposition concernant le bassin méditerranéen, régions subsahariennes comprises (Quezel \& SAnta, 1963; FeinbrunDothan, 1978; Qaiser, 1979; Pottier-Alapetite, 1981; Mouterde, 1983; Boulos, 1999). Des comparaisons en herbier à $\mathrm{P}$ ont aussi été faites pour l'ensemble des espèces d'Echium circumméditerranéens disponibles.

\section{Taxonomie}

Echium montenielluense Delage, spec. nova (fig. 1, 2).

Holotypus: France. Corse: Capicorsu, Cne de SantaMaria-di-Lota, versant S du Monte Niellu, $965 \mathrm{~m}$, 22.V.2017, Delage s.n. (Hb. CBNC!; iso-: G [G00341786]!, $\mathrm{P!})$.

Echium montenielluense Delage is similar to E. anchusoides Bachetta et al. from which it differs by its wider leaves, obovate to lanceolate, its inflorescence fairly often ramified with longer branches and its non versicolor bright pink corolla.

Hémicryptophyte vivace, en rosette, prostrée, hispide dans toutes ses parties; souche peu ou pas lignifiée, habituellement simple, très rarement ramifiée, à racine pivotante verticale dépassant $10 \mathrm{~cm}$. Indument des tiges, feuilles, bractées et calices dimorphe, composé d'une part de poils simples, courts, denses et appliqués et d'autre part de poils épineux, dressés, atteignant 3,7(-4) mm de long, à base tuberculeuse pluricellulaire. Tiges peu nombreuses, généralement 2 à 4 , rarement jusqu'à 7 , exceptionnellement 12 , habituellement prostrées, parfois prostrées-ascendantes, de $5-16(-30) \mathrm{cm}$ de long, naissant latéralement de la rosette, densément couverte de poils 


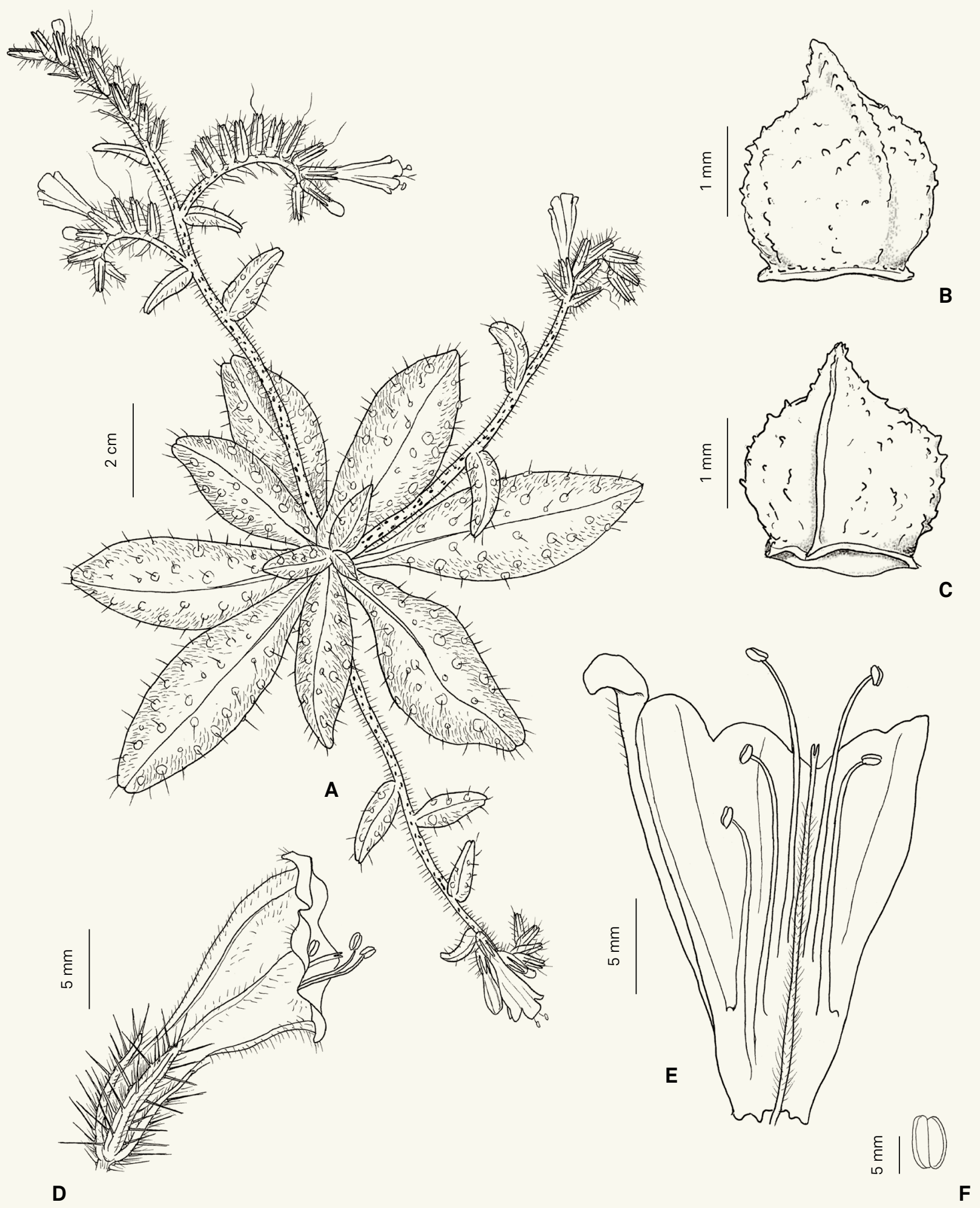

Fig. 1. - Echium montenielluense Delage. A. Inflorescence et rosette; B. Méricarpe (face dorsale); C. méricarpe (face ventrale); D. Détail de la fleur à l'anthèse; E. Corolle étalée après ouverture dorsale; F. Anthère.

[Dessins: A. Delage] 
courts $(0,4-0,8 \mathrm{~mm})$ appliqués, rétrorses et de poils tuberculeux dressés (1,9-3,6 mm) portés par des pustules rouges. Feuilles basales obovales à lancéolées, subsessiles ou atténuées en pétiole court par décurrence du limbe, formant une rosette peu dense, présente à l'anthèse; Limbe de $28-65(-72) \times(7-) 8$ $-17(-22) \mathrm{mm}$, très hispide, portant de nombreux poils tuberculeux dressés $1,2-3,7(-4) \mathrm{mm}$, paraissant vert grisâtre par un indument dense de poils courts $(0,3-0,8 \mathrm{~mm})$ appliqués antrorses; Présence de nombreuses pustules claires. Marges entières et droites. Feuilles caulinaires (4 à 6) lancéolées, sessiles, hispides et pustuleuses; limbe de 16-26(-33) × 4-9(-11) $\mathrm{mm}$. Inflorescence en cyme scorpioïde majoritairement simple, assez fréquemment ramifiée une fois, rarement ramifiée deux fois, voire trois à six fois; ramifications de taille variable, de 2 à $15,5 \mathrm{~cm}$, parfois réduites à un glomérule de quelques fleurs; cymes pauciflores à 4-19(-36) fleurs sessiles; bractées un peu plus courtes que le calice, hispides, étroitement lancéolées, de 5-10 × 1.5-2 mm. Calice divisé jusqu'à la base en 5 lobes étroitement lancéolés-linéaires, très hispides (poils de 1,8-3,2 mm), de taille à peu près égale, de $8-11(-12) \times 1-1,8 \mathrm{~mm}$ à l'anthèse; corolle nettement zygomorphe, assez étroitement infundibuliforme, de 19-22(-24) mm en longueur dorsale, assez densément et régulièrement pubescente, sauf à la base du tube, toujours d'un rose vif (très rarement rose pâle, couleur observée chez un seul individu), plus pâle sur les nervures soulignées de rose foncé (couleur de la corolle invariable, rose dès le bouton et jusqu'à l'anthèse, ne devenant bleue qu'au flétrissement et à la dessiccation); tube plus pâle, avec un anneau basal de 10 petites écailles portant chacune $1-2$ poils $(<0,3 \mathrm{~mm})$ translucides. Etamines de différentes longueurs: la dorsale et les latérales incluses, insérées à $7-8 \mathrm{~mm}$ de la base de la corolle, à filet de $7 \mathrm{~mm}$ pour la première, de $9 \mathrm{~mm}$ pour les suivantes; les deux ventrales sortant nettement du tube, insérées à 9-10 mm de la base de la corolle, à filet de 9-10 mm; filets incurvés, glabres, roses; anthères assez largement elliptiques, de $1,5 \times 1 \mathrm{~mm}$, d'un violet pâle. Style de 18-22 mm de long, rose à blanc-rosé, assez densément couvert de poils blancs, sauf à l'extrémité. Stigmate bifide, à extrémité violet pâle. Méricarpes de 2,6-2,8 × $2 \mathrm{~mm}$, ovoïdes-trigones, aigus, grossièrement et irrégulièrement verruqueux, brun sombre, à tubercules et carènes d'un brun clair; face dorsale très bombée, carénée sur $2 / 3$ de sa longueur, face ventrale carénée sur toute sa longueur.

Etymologie. - Cette espèce est nommée d'après son unique localité connue à ce jour, au Monte Niellu.

Distribution, écologie et phénologie. - Echium montenielluense est vraisemblablement endémique de Corse et n'est pour l'instant connu que d'une seule localité, dans le Capicorsu, sur le versant sud du Monte Niellu, au-dessus de Santa-Maria-diLota. Toutefois un Echium indéterminé observé dans une localité proche par Y. Petit (CBNC) et appartenant vraisem- blablement à cette espèce devra être retrouvé, son identité et sa localisation précisées.

La station est située sur un versant très pentu constitué de barres rocheuses et d'éboulis de serpentinite, entre 900 et $1030 \mathrm{~m}$ d'altitude (fig. 2F). Tous les individus actuellement connus sont circonscrits dans une surface d'environ 6 ha. Ils sont disséminés dans la localité en petites colonies comptant au maximum quelques dizaines d'individus. Les recensements que nous avons effectués le 31 mai et le 11 juillet 2017 montrent un effectif d'environ 250 individus (221 plantes comptées, se répartissant en 79 pieds fleuris ou fanés et 142 rosettes non florifères à des stades de développement variés). Les pieds en fleur ont été observés en mai et juin.

Les plantes poussent dans les ouvertures très peu végétalisées des fruticées naines à Euphorbia spinosa L. et Genista salzmannii DC., que l'on peut assimiler au Genisto salzmannii-Alyssetum robertiani Ro. Molinier 1959, même si Alyssum robertianum Bernard et al. n'y est que très ponctuel. En dehors des espèces déjà citées, la formation est constituée de: Scrophularia canina var. deschatresii Gamisans, Anthyllis hermanniae L., Cistus salviifolius L., Thymus herba-barona Loisel., Helichrysum italicum (Roth) G. Don subsp. italicum, Stachys glutinosa L., Rosa serafinii Viv., Teucrium capitatum L. subsp. capitatum, Dianthus arrostii C. Presl (= D. sylvestris subsp. longicaulis auct.), Cerastium boissierianum Greuter \& Burdet, Galium lucidum All. et Carlina corymbosa L.

Dans la fruticée, Echium montenielluense semble privilégier les accumulations gravelo-terreuses au sein des rocailles (fig. 2G), où il est accompagné par les espèces suivantes: Reichardia picroides (L.) Roth, Sedum album L., Sedum dasyphyllum L., Tuberaria guttata (L.) Fourr., Crepis foetida L., Vulpia myuros (L.) C.C. Gmel., Hypochaeris achyrophorus L., Dactylis glomerata subsp. hispanica (Roth) Nyman, Linum trigynum L. et Brachypodium bybridum Catalàn et al.

On peut également observer l'espèce, quoique plus rarement, dans les fissures des petites parois rocheuses et des éboulis instables quasi verticaux, avec une végétation vestigiale.

Notes. - Avec des similitudes morphologiques plus ou moins marquées, il apparaît que la nouvelle espèce se rapproche morphologiquement d'E. anchusoides, E. sabulicola et E. creticum. Le tableau 1 présente les caractères discriminants entres ces trois espèces.

Echium montenielluense se distingue aisément d'E. creticum par son port prostré, ses dimensions bien moindres en conditions normales de végétation, son inflorescence beaucoup plus simple, la taille et la couleur de ses fleurs, ses étamines à filet toujours glabre. Il présente d'avantage de ressemblances avec $E$. sabulicola, avec lequel il partage un port prostré à ascendant. Il s'en distingue cependant par l'indument appliqué rétrorse des tiges (vs antrorse), l'inflorescence simple ou peu ramifiée (vs paniculiforme et nettement ramifiée), les feuilles basales plus 

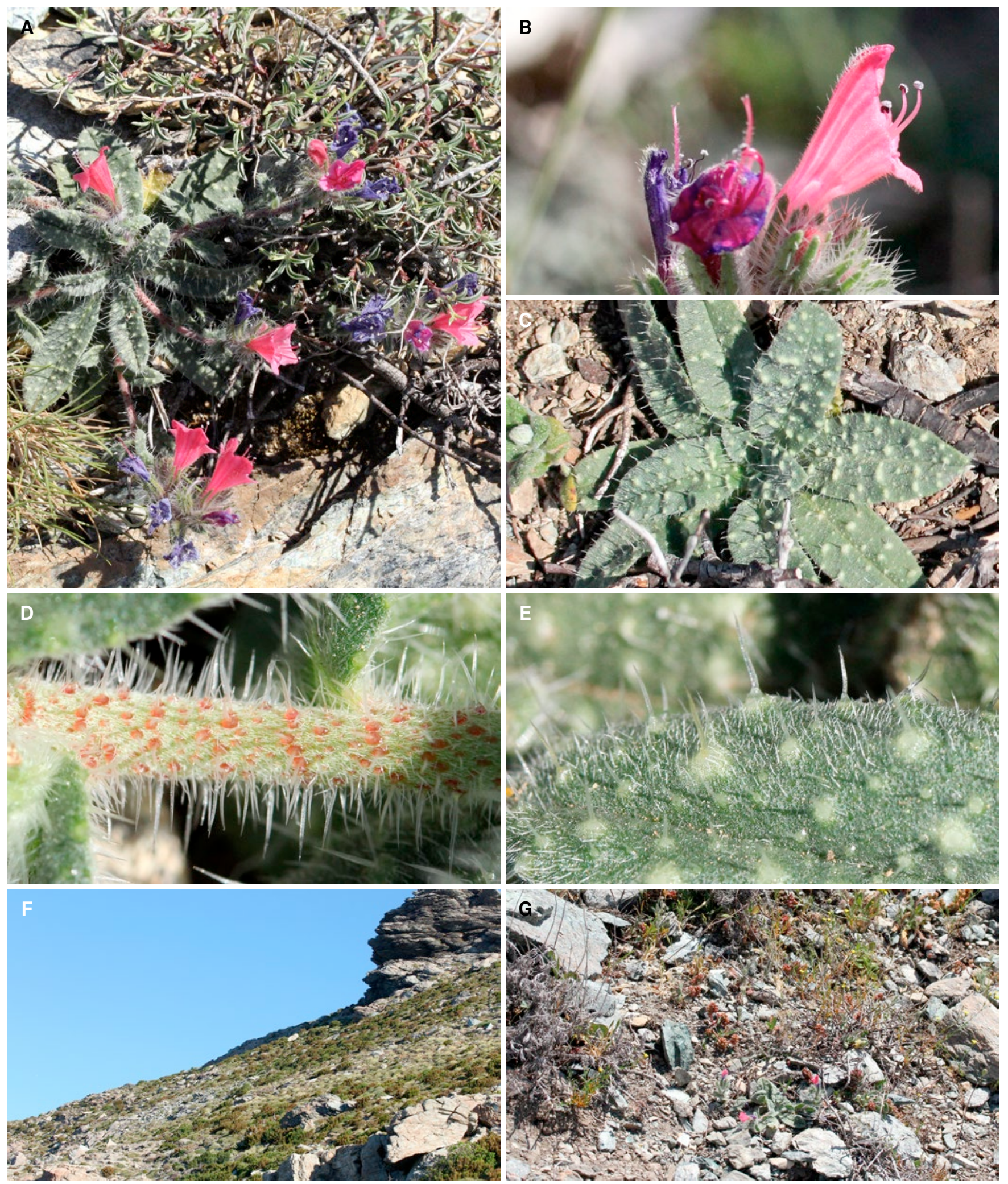

Fig. 2. - Echium montenielluense Delage. A. Inflorescence et rosette; B. Détail d'une cyme; C. Rosette; D. Détail de l'indument de la tige; E. Détail de l'indument du limbe; F. Vue générale de l'habitat au Monte Niellu; G. Détail de l'habitat.

[Photos: A. Delage] 
petites, ne dépassant généralement pas $6,2 \mathrm{~cm}$ (vs 9,5-15 cm), les fleurs toujours roses (vs bleu violet).

Echium montenielluense est proche morphologiquement d E. anchusoides mais s'en distingue par les dimensions et la forme des feuilles et en particulier le rapport $1 / \mathrm{L}$, la fréquence des ramifications dans l'inflorescence et la couleur des fleurs invariablement rose vif. De plus, la face supérieure du limbe est parsemée de grosses pustules claires très nettes, qui sont peu frappantes sur les plantes sardes que nous avons observées in situ. L'indument est plus dense et plus hérissé chez E. montenielluense, y compris sur les tiges, lui conférant un aspect grisâtre et épineux marqué.

Les plantes sont aussi nettement séparées géographiquement, E. montenielluense étant isolé sur la crête du Capicorsu dans le nord de la Corse, la station la plus proche d'E. anchusoides se trouvant dans l'archipel de la Maddalena, dans les bouches de Bunifaziu. Elles ont également une écologie différente, E. anchusoides poussant du littoral jusqu'à $1800 \mathrm{~m}$, sur des pentes rocailleuses, des pelouses ouvertes et subnitrophiles, sur des sols siliceux variés (granite et autres), E. montenielluense semblant être inféodé aux fruticées naines ouvertes sur éboulis de serpentinite (roche à notre connaissance absente de Sardaigne), vers 1000 m d'altitude.

Paratypi. - France. Corse: Capicorsu, Santa Maria di Lota, versant S du Monte Niellu, fruticée naine sur éboulis de serpentinite, 980 m, 23.V.2018,
Delage s.n. (Hb. CBNC); ibid. loco, 980 m, 23.V.2018, Delage, s.n. (G); ibid. loco, 980 m, 23.V.2018, Delage s.n. (G).

\section{Remerciements}

Nous tenons à remercier le personnel du Muséum National d'Histoire Naturelle de Paris, en particulier le Prof. Serge Muller, responsable scientifique de l'herbier national, pour ses conseils dans nos démarches, et le Dr. Germinal Rouhan, conservateur de l'herbier des fougères et des lycophytes, pour son sympathique accueil, ses indications et sa disponibilité pendant notre consultation. Nous sommes particulièrement reconnaissants au Prof. Daniel Jeanmonod pour sa relecture critique, ses corrections, ses nombreux conseils et ses observations concernant les échantillons d'E. anchusoides conservés à Genève. Nous souhaitons également remercier, pour leurs conseils, leur relecture critique et leurs corrections, le Prof. Guilhan Paradis et nos collègues Carole Piazza et Christophe Panaïotis ainsi que les éditeurs de Candollea.

Tableau 1. - Morphologie comparée de Echium montenielluense Delage avec E. anchusoides Bacheta et al., E. creticum L. et E. sabulicola Pomel. Les valeurs ou caractères discriminants sont notés en caractères gras.

\begin{tabular}{|c|c|c|c|c|}
\hline Caractères & E. montenielluense & E. anchusoides & E. sabulicola & E. creticum \\
\hline Port des tiges & prostré à prostré-ascendant & prostré à prostré-ascendant & décombant à ascendant & dressé \\
\hline Taille des tiges $[\mathrm{cm}]$ & $5-16(-30)$ & $5-30(-40)$ & $15-60$ & $25-90$ \\
\hline Pilosité appliquée (tiges) & rétrorse & rétrorse & antrorse & rétrorse \\
\hline Forme des feuilles basales & lancéolées à obovales & $\begin{array}{l}\text { linéaires } \\
\text { à étroitement oblancéolées }\end{array}$ & oblancéolées & $\begin{array}{l}\text { ovales } \\
\text { à ovales-lancéolées }\end{array}$ \\
\hline Longueur des feuilles [cm] & $2,8-6,5(-7,2)$ & $2,5-7$ & $2,1-9,5(-15)$ & $2,5-13(-18)$ \\
\hline Largeur des feuilles $[\mathrm{cm}]$ & $0,7-1,7(-2,2)$ & $0,4-0,8$ & $0,4-1,5(-2)$ & $0,5-2(-2,5)$ \\
\hline $\mathrm{I} / \mathrm{L}$ & $0,19-0,50$ & $0,11-0,16$ & - & - \\
\hline Inflorescence & $\begin{array}{l}\text { cymes en majorité simples, } \\
\text { assez fréquemment une } \\
\text { ramification, parfois plusieurs } \\
\text { (jusqu'à 6); ramifications } \\
\text { de longueur variable }\end{array}$ & $\begin{array}{l}\text { cymes simples ou rarement } \\
\text { une courte ramification }\end{array}$ & $\begin{array}{l}\text { inflorescence ramifiée, } \\
\text { paniculiforme ou simple } \\
\text { à cymes géminées }\end{array}$ & $\begin{array}{l}\text { cymes lâches groupées } \\
\text { en inflorescence } \\
\text { paniculiforme }\end{array}$ \\
\hline Taille de la corolle $[\mathrm{mm}]$ & $19-20(-24)$ & $16-20$ & $14-20(-25)$ & $(17-) 20-35(-40)$ \\
\hline Couleur de la corolle & rose vif & $\begin{array}{l}\text { rose-carmin } \\
\text { devenant bleu-violet }\end{array}$ & bleu-violet & $\begin{array}{l}\text { rouge } \\
\text { à rougeâtre-pourpre }\end{array}$ \\
\hline Anneau basal de la corolle & $\begin{array}{l}10 \text { écailles portant } \\
1-2 \text { trichomes }\end{array}$ & $\begin{array}{l}10 \text { écailles portant } \\
5-10 \text { trichomes }\end{array}$ & non précisé & non précisé \\
\hline Filet des étamines & glabre & glabre & $\begin{array}{l}\text { glabre } \\
\text { ou faiblement scabre }\end{array}$ & poilu (rarement glabre) \\
\hline
\end{tabular}




\section{Références}

Arrigoni, V. (2013). Fl. Isola Sardegna 4: 243-255.

Bachetta, G., S. Brullo \& F. Selvi (2000). Echium anchusoides (Boraginaceae), a new species from Sardinia (Italy). Nordic J. Bot. 20: 271-278.

Boulos, L. (1999). Fl. Egypt 1: 302-308.

Briquet, J. \& R. de Litardière (1955). Prodr. Fl. Corse 3/2.

Feinbrun-Dothan, N. (1978). Ericaceae to Compositae. In: Zohary M. (ed.), Fl. Palaest. 3: 74-77.

Fürnkranz, D. (1982). Echium L. In: Pignatti, S. (ed.), Fl. Italia 2: 405-407.

Gib bs, P.E. (1971). Taxonomic studies on the genus Echium. I. An outline revision of the Spanish species. Lagascalia 1: 27-82.

Gibbs, P.E. (1972). Echium L. In: Tutin, T.G. et al. (ed.), Fl. Eur. 3: 97-100.

Jeanmonod, D. \& J. Gamisans (2013). Flora Corsica. Ed. 2. Bull. Soc. Bot. Centre-Ouest num. spéc., 39.

Lambinon, J. \& R. Deschâtres (1987). Echium creticum L. subsp. creticum. Candollea 42: 53.

Mouterde, S.J. (1983). Nouv. Fl. Liban \& Syrie 3: 74-77.

Pottier-Alapetite, G. (1981). Fl. Tunisie: 749-756.

Quezel, P. \& S. Santa (1963). Nouv. Fl. Algérie 2: 762-766.

QAISER, M. (1979). Boraginaceae. In: Ali, S.I. \& S.M.H.JAFri (ed.), Fl. Lybia 68: 33-50.

Tison, J.-M. \& B. de Foucault (2014). Flora Gallica. Flore de France. Biotope Editions.

Tison, J.-M., P. Jauzein \& H. Michaud (2014). Flore de la France méditerranéenne continentale. Naturalia Publications.

VALDÉs, B. (2007). Notas sobre el genéro Echium L. Lagascalia 27: 53-71.

Valdés, B. (2012). Echium L. In: Talavera, S. et al. (ed.), Fl. Iber. 11: 413-446.

VAlsecchi, F. (1977). Contributo alla conescenza del genere Echium: I - Echium della Sardegna. Webbia 32: 101-127. 